\title{
Reflexionsseismische Messungen in Fließgewässern und Stauräumen
}

\author{
Erwin Heine · Marina Golja · Helmut Habersack · Christoph Hauer
}

Online publiziert: 12. Mai 2020

(C) Der/die Autor(en) 2020

\begin{abstract}
Zusammenfassung Informationen über den Internaufbau der oberen Sedimentschichten sind sowohl für aktuelle Monitoring-Maßnahmen als auch für prognostische Prozessmodellierungen des Sedimenttransports von großer Bedeutung. Seismische Verfahren bieten hier eine sehr gute Möglichkeit zur linienhaften Erfassung dieser Grundlagendaten. Ihre Anwendbarkeit ist jedoch

SBP bei Feinsedimentböden schließen, Analysen zeigen allerdings auch Anwendungsgrenzen im Kiesbereich auf. Zumindest bei Böden mit geringer Kiesdeckschicht finden sich aber spezielle Anwendungsmöglichkeiten und so gibt die Summe der bisherigen Erkenntnisse Anlass für weiterführende Untersuchungen mit SBPs bzw. alternativen seismischen Methoden.
\end{abstract} eine Frage der vorliegenden Objekteigenschaften (Wassertiefe, Strömung etc.) und die Qualität der Ergebnisse, wie Eindringtiefe und Auflösung der Sedimentstrukturen, wird stark von systemspezifischen Parametern und Sedimentbeschaffenheit beeinflusst. Dieser Beitrag behandelt den Einsatz von zwei hochauflösenden und kompakten Seeseismik-Systemen zur Detektion des Sedimentaufbaus in Gewässern mit grundsätzlich unterschiedlichem Sohlaufbau. Mit den parametrischen Sub-Bottom-Profilern (SBP) der Firmen Innomar und Kongsberg Maritime wurden umfangreiche Sedimentecholotungen des Absetzbeckens von Rottau (Schluff) sowie an zwei Teilabschnitten der Donau (Kies) durchgeführt. Ein Vergleich von Sedimentkernauswertungen mit den resultierenden Echogrammen lässt auf das große Potenzial von

\author{
Ass.-Prof. DI Dr. E. Heine $(\bowtie)$ \\ Department für Raum, Landschaft \\ und Infrastruktur, Institut \\ für Geomatik, Universität \\ für Bodenkultur Wien \\ Peter-Jordan-Straße 82, 1190 Wien, \\ Österreich \\ erwin.heine@boku.ac.at
}

\section{Golja .}

Univ.-Prof. DI Dr. H. Habersack .

PD DI Dr. C. Hauer

Christian Doppler Labor

für Sedimentforschung

und -management, Institut

für Wasserbau, Hydraulik

und Fließgewässerforschung,

Universität für Bodenkultur Wien,

Muthgasse 107, 1190 Wien, Österreich
Schlüsselwörter Hydrografische Vermessung · Reflexionsseismik . Seismic Profiling - Sub-BottomProfiling · Sedimentecholotung • Sedimentkerne $\cdot$ Flusssohle .

\section{Seismic reflection profiling on rivers and reservoirs}

Abstract Information about the internal structure of the upper sediment layers is of great importance both for current monitoring measures and for prognostic process modeling of sediment transport. Seismic methods offer a sophisticated approach to determine this basic data, provided that object properties such as water depth, current, etc. are suitable. The quality of the results, like penetration depth and resolution of the sediment structure depends on the system-specific parameters and the sediment quality. This article deals with the use of high-resolution seismic systems in order to detect sediment build-up in water bodies with fundamentally different soil structures. Parametric sub-bottom profilers (SBP) from Innomar (Germany) and Kongsberg Maritime (Norway) were used to carry out extensive sediment echo sounding in the sedimentation basin of Rottau (silt) and the river Danube (gravel). Comparing the evaluation of sediment cores to the resulting echograms indicates a great potential of SBP for fine sediment soils, but also shows limitations where coarser material occurs. Some special application options for soils with a thin top layer of gravel are Staubecken · Donau available though, thus initiating further investigations with SBPs and alternative seismic methods for this type of sediments.

Keywords hydrography $\cdot$ reflexion seismic $\cdot$ seismic profiling $\cdot$ SubBottom-Profiling · sediment echo sounding · sediment coring - river bed . storage lake $\cdot$ Danube river

\section{Einleitung}

Die Ablagerung von Sedimenten in Stauräumen von Speicher- und Laufkraftwerken bildet wesentliche Herausforderungen für den Betrieb der Anlagen sowohl aus technischen, ökonomischen, aber auch ökologischen Gesichtspunkten (Bock et al. 2019; Hauer et al. 2018). Beobachtungen von Verlandungsraten bzw. auch die Effizienz von Maßnahmen (z.B. Spülungen) wurden seit Beginn der Errichtung vor allem im Bereich der Großwasserkraft basierend auf Stauraumvermessungen kontinuierlich durchgeführt (Dang et al. 2010; Chapman 1996; Mahmood und Mundial 1987). Die durchgeführten Analysen und Auswertungen bezogen sich vor allem auf Ablagerungs- und Erosionsvolumina und -flächen (De Araujo et al. 2006; Schleiss et al. 2016; Foster und Walling 1994). Analysen über die Sedimentzusammensetzung (Korngrößen, Lagerungsdichte) konnten nur punktuell (Kolbenlotmessungen, Freeze-Cores) gewonnen werden (McIntyre 1993; Navas et al. 2009).

Für Optimierungsfragen der Speicherbewirtschaftung besteht aber durchaus die Notwendigkeit, diese Informationen flächendeckend und in einer hohen Auflösung zu erlangen (Hauer et al. 2018). Dies betrifft vor allem auch die Verwendung von Prognosewerkzeugen für Vorhersagen der Wirkung von Maßnahmen zur Verbesserung des Sedimentmanagements, wobei aufgrund der fortschreitenden Entwicklungen in der Numerik mehrdimensionale Modellierungen von Abfluss- und Sediment- 
transport die Prozesse bereits hochauflösend abbilden können (Haun et al. 2013; Faghihirad et al. 2015).

Als Modellierungsgrundlagen werden hier jedoch oft punktbasierte und in der Folge flächig aggregierte Informationen von Sedimentzusammensetzungen in Stauräumen gewählt (Tritthart et al. 2020). Ziel dieses Fachbeitrags ist es, für diesen speziellen Teil der Grundlagenerhebung und Aufbereitung zu einer optimierten Stauraumbewirtschaftung den aktuellen Stand der Forschung zur Erfassung der Sedimentzusammensetzungen zu präsentieren. Es werden verschiedene Systeme zur hochauflösenden Stauraumvermessung vorgestellt, auch mit zusätzlichen technischen Möglichkeiten zur Detektion der Sedimentzusammensetzung. Anhand einiger ausgewählter Fallbeispiele wird auch deren Anwendbarkeit getestet bzw. werden weiterführende wissenschaftliche Untersuchungen diskutiert.

\section{Hydrografische Vermessung}

Die hydrografische Vermessung (engl. Hydrography) befasst sich mit Erfassung, Auswertung, Modellierung und Visualisierung der Gestalt, der Beschaffenheit und des Aufbaus von Gewässersohlen. Um detaillierte morphologische Untersuchungen der Sohle von Gewässern durchzuführen und um eine geologische Modellvorstellung über die Sedimente in wenigen Metern Tiefe unter der Gewässersohle zu entwickeln, ist die Kombination von detaillierten topografisch/bathymetrischen Daten und weiteren geophysikalischen Messdaten des Gewässersohlenuntergrunds notwendig (Hilbe et al. 2011; Heine et al. 2016; Schnabel et al. 2014).

Dabei werden für die Aufnahme der Topografie der Gewässersohle hochauflösende Fächerecholotsysteme eingesetzt, während geophysikalische Informationen über den Aufbau der darunterliegenden Sedimentschichten mittels hochauflösender seismischer Systeme erhoben werden. Um das seismische Abbild des Untergrunds verlässlich zu interpretieren, werden an ausgewählten Stellen zusätzliche Informationen aus Bohrkernen zur Interpretation der seismischen Daten hinzugezogen.

\subsection{Fächerecholotung der} Gewässersohle

Gegenüber herkömmlichen Einzelstrahlecholotmessungen ohne Sensorbewegungskompensation und häufig mit Profilabständen von mehreren Zehnermetern, gewannen in den letzten Jahren genauere und höher auflösende Messsysteme wie Fächerecholote zunehmend an Bedeutung für die Untersuchung von Sedimentationsprozessen in Stauräumen und Fließgewässern (Baran et al. 2013; Buchta 2019). Diese dienen als Lieferant von abiotischen Inputdaten (DTMs) für die numerische Modellierung (Tritthart et al. 2012), ökohydraulische Untersuchungen (Hauer et al. 2012) und Analysen zu Feststoffhaushalt und Morphodynamik (Habersack et al. 2012). Besonders hohe Genauigkeitsanforderungen ergeben sich hier beim Monitoring von Sedimentablagerungen in kritischen Gewässerabschnitten, wie Seichtstellen für die Schifffahrt bzw. renaturierten Flussabschnitten (Liedermann et al. 2016).

Vermessungs-Fächerecholote (Multi Beam Echo Sounder, MBES) „scannen“ die Gewässersohle mit bis zu 512 Messstrahlen und sind in der Lage, detaillierte Geländemodelle mit Höhengenauigkeiten von wenigen Zentimetern zu liefern. Dazu ist es aber erforderlich, dass sowohl das Messsystem mit all seinen Sensoren als auch der Messprozess die dafür vorgesehenen hohen Qualitätsstandards erfüllen (Heine 2017). Die hier eingesetzten Messsysteme der VERBUND Hydro Power GmbH beziehungsweise der via donau - Österreichische Wasserstraßen-Gesellschaft $\mathrm{mbH}$ wurden als Gesamtsystem vom Fächerecholothersteller Kongsberg Maritime für den hochgenauen Vermessungseinsatz im Flachwasser konfiguriert und auch mit entsprechend hochwertigen externen Sensoren ausgestattet.

So verfügen derartige Echolote über eine dynamische Fokussierung der Messstrahlen, wodurch durch Berücksichtigung der Messdistanz die Einflüsse der kugelförmigen Schallwellenausbreitung auf die Distanzmessgenauigkeit im Flachwasser erhöht werden. Durch die Integration eines hochwertigen Inertialmesssystems mit einer Bewegungsdetektionsgenauigkeit besser als $\pm 0,1^{\circ}$ werden Stampf- und Rollbewegungen des Messboots dynamisch kompensiert. Zur Bestimmung der systematischen Einbauwinkel-Feh- ler (Roll, Stampf und Kurs) des Fächerecholotschwingers im Verhältnis zum GNSS/INS-System sowie einer Latenz in der Positionierung wird jeweils vor Beginn der Messkampagne eine Feldkalibrierung, der sogenannte Patch-Test durchgeführt. Für die unmittelbare Tiefenmessgenauigkeit ist in erster Linie die Kenntnis der Schallgeschwindigkeit $(\sim 1500 \mathrm{~m} / \mathrm{s})$ entlang des Echolotstrahls entscheidend. Um die Fehler der Schallgeschwindigkeit zu verhindern bzw. zu minimieren, erfolgt einerseits eine permanente Messung der Schallgeschwindigkeit nahe dem MBES-Schwinger, andererseits wird zeitlich und räumlich verteilt gemessen. Dies erfolgt an repräsentativen (tiefen) Stellen im Messgebiet mit einer SchallgeschwindigkeitsSonde (SVP). Die Geschwindigkeitswerte werden entlang von vertikalen Profilen - beginnend an der Wasseroberfläche bis zur Sohle - aufgenommen und in der Software für die Datenerfassung bzw. Auswertung an die jeweiligen Echolotdaten angebracht (Heine 2017).

Zur Erhöhung der Detailgenauigkeit werden die die Gewässersohle abtastenden Fächerlotmessstreifen mit mindestens 50\%iger Überlappung zwischen den benachbarten Streifen gefahren. Dadurch wird die Topografie gleich mehrfach und aus verschiedenen Strahlrichtungen abgetastet. Neben der hochgenauen bathymetrischen Vermessung ermöglichen derartige MBES-Systeme auch die Aufzeichnung der Rückstreustärke sowohl in der Wassersäule als auch von der Gewässersohle selbst. Die winkelabhängige Rückstreuintensität der Sohle ist charakteristisch je nach Beschaffenheit des Untergrunds, wie etwa Sand, Kies und Schlick. Daraus abgeleitete Intensitätskarten stellen eine bedeutende Informationsquelle für geomorphologische Studien alpiner Seesedimentationsprozesse dar (Strasser et al. 2020).

Das aus den Fächerecholotmessungen generierte exakte morphologische Abbild der Gewässersohle findet aber nicht nur Eingang als wesentliche Grundlage für die numerische Modellierung, sondern ist insbesondere ein essenzieller komplementärer Datensatz bei der Aufnahme, Analyse und Interpretation reflexionsseismischer Messdaten. So werden etwa Geländeneigungsinformationen zur optimalen Ausrichtung bzw. Energieverstärkung des Schallkegels von Sedimentecholoten benötigt (vgl. Abb. 4b). 


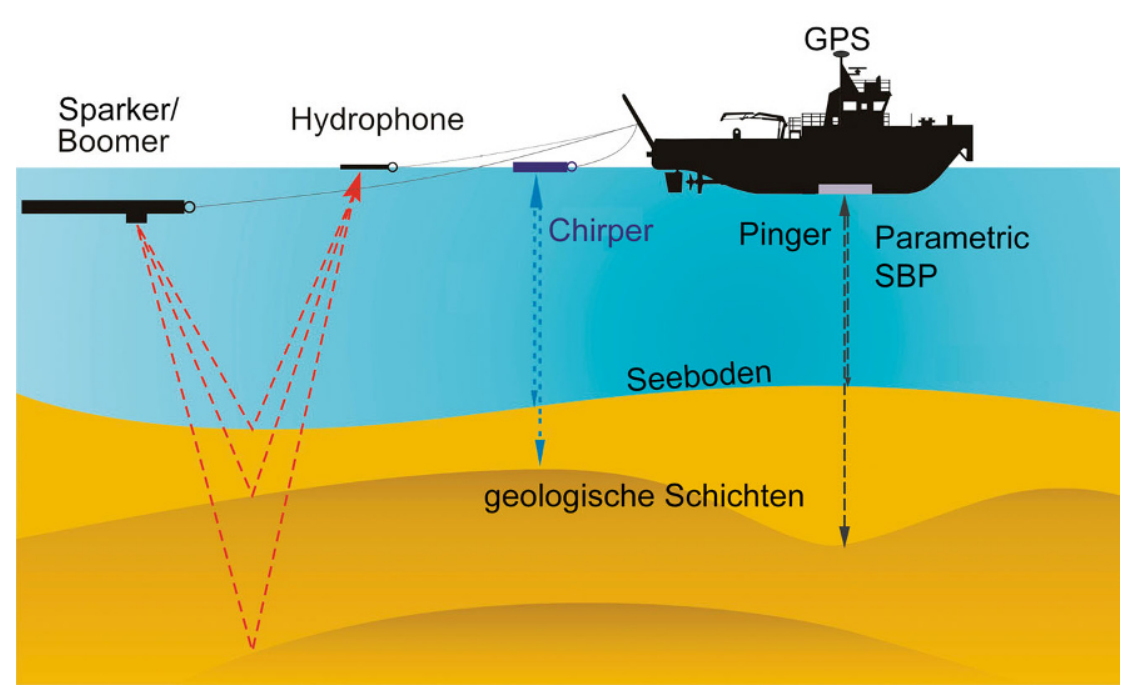

Abb. 1 Prinzip des Einsatzes von Seeseismik-Systemen. (Nach Stoker et al. 1997)

\subsection{Reflexionsseismische Messverfahren}

\subsubsection{Messprinzip und Systemeinteilung}

Oberflächennahe zweidimensionale Reflexionsseismik ist eine geeignete geophysikalische Methode zur hochauflösenden Kartierung komplexer geologischer Strukturen in Gewässerböden (Schnabel et al. 2014). „Seismic Profiling“ oder genauer „Seismic Reflection Profiling“ ist den Verfahren der Reflexionsseismik zuzuordnen. Dabei werden seismische Wellen künstlich erzeugt, und zur Bestimmung von Schichtgrenzen im Sohluntergrund (sub-bottom) eingesetzt. Die Eindringtiefe hängt dabei von der Frequenz, dem geologischen Aufbau und der eingesetzten Schallenergie ab (Missiaen et al. 2008).

Bei der seismischen Messung auf der freien Wasseroberfläche, der sogenannten Seeseismik, stehen für die künstliche Anregung von seismischen Wellen unterschiedliche Systeme zur Verfügung (Abb. 1 und Tab. 1). Dabei wird Schallenergie in Form eines kur- zen Impulses Richtung Gewässersohle ausgesandt. Die Schallenergie wird von der Sohloberfläche und von den darunterliegenden Sedimentschichten reflektiert. Die reflektierte Energiemenge hängt dabei von der Dichte bzw. dem Unterschied („Kontrast“) der akustischen Impedanz (Produkt aus Dichte und Schallgeschwindigkeit) der Sedimentschichten ab. Das reflektierte Signal durchläuft dann den Wasserkörper und wird an den Empfängern aufgezeichnet. Bei den Empfängern handelt es sich je nach Messsystem um einen Transducer oder geschleppte Hydrophone.

Jene Systeme, welche die Schallquelle und die Hydrophone an einem Seil hinter der Schallquelle schleppen („streamer“) sind aufgrund der sehr eingeschränkten Manövrierbarkeit für einen Einsatz auf kleinen Stauräumen oder Wasserstraßen (Donau) nicht oder nur sehr bedingt geeignet. Des Weiteren ist es im Falle von geringen Wassertiefen und bewegter Wasseroberfläche (Strömung, Wellengang) schwierig, qualitativ hochwertige Aufnahmen mit
„streamer“-Messsystemen zu gewinnen, da es hierbei zu unerwünschter Überlagerung zwischen den empfangenen Reflexionswellen und jenen des ausgesandten Signals kommen kann.

Transducer-Systeme sind meist kompakt gebaute Sub-Bottom-Profiler-Systeme, bei denen Schallquelle und Empfängerteil kompakt in einer TransducerEinheit zusammenfasst sind, welche entweder direkt am Schiffsrumpf montiert oder zur Reduktion von schiffsbezogenen Störeinflüssen auf einer eigenen kleinen Plattform (Minikatamaran) in kurzem Abstand vom Schiff vertäut ist. Hierbei ist auch eine exakte Georeferenzierung der Messdaten durch GNSS-basierte Positionierung des Transducers gegeben.

\subsection{Parametrische Sub-Bottom-Profiler}

Im Rahmen des Christian Doppler Labors für Sedimentforschung und -management wurde an zwei Gewässern (Speicher Rottau und Donau östlich von Wien) mit stark unterschiedlichem geologischen Sohlaufbau die Leistungsfähigkeit von zwei parametrischen SubBottom-Profilern getestet. Dabei handelt es sich um das SES-2000 compact der Firma Innomar sowie um das TOPAS PS 120 der Firma Kongsberg. Beide sind portable Systeme, welche ohne großen Aufwand außen am Schiffsrumpf montiert werden können. Die technischen Spezifikationen der beiden Systeme sind sehr ähnlich und somit vergleichbar. So liegt der Einsatzbereich bei Wassertiefen zwischen 1 und $400 \mathrm{~m}$, und die maximale Eindringung in den Untergrund liegt, je nach Bodenbeschaffenheit, im Bereich einiger Zehnermeter.

Beide Systeme nutzen den parametrischen Effekt, um ein eng gebündeltes, tieffrequentes akustisches Signal mit hoher räumlicher Auflösung $\mathrm{zu}$ erzeugen, welches in der Lage ist, in den Untergrund einzudringen und die oberen Sedimentschichten $\mathrm{zu}$,kar-

Tab. 1 Akustische Eigenschaften von Seeseismik-Systemen. (Nach Ramsay 2017)

\begin{tabular}{|l|l|l|l|l|}
\hline System & Frequenz (in kHz) & Schallquelle \& Empfänger & Auflösung (in m) \\
\hline Chirp SBP & $2-16$ & Frequenzmoduliert; Transducer & $0,05-0,1$ & Eindringtiefe (in m) \\
\hline Parametric SBP & $2-22$ & Parametrisch; Transducer & $0,05-0,1$ & $5-50$ \\
\hline Pinger SBP & $2-12$ & Transducer & 0,2 & $10-50$ \\
\hline Bubble Pulser & 0,4 & Platte \& geschleppte Hydrophone & $0,3-0,5$ & $20-100$ \\
\hline Boomer & $0,3-6$ & Spulenplatte \& geschleppte Hydrophone & $0,2-0,5$ & $20-150$ \\
\hline Sparker & $0,2-3$ & Elektroden \& geschleppte Hydrophone & $0,3-1$ & $30-750$ \\
\hline Mini-Airgune & $0,1-3$ & Luftkanone \& geschleppte Hydrophone & $0,5-1$ & $30-200$ \\
\hline
\end{tabular}


tieren“. Je nach Sedimentzusammensetzung, Lagerungsdichte und Gasanteil im Untergrund wird das Signal an den Schichtgrenzen total oder teilweise reflektiert, sodass ein Teil der Welle weiter eindringt und am nächsten Reflexionshorizont wieder total oder teilweise reflektiert wird usw. Die zurückkommenden Reflexionen werden am Bordrechner als Rohdaten abgespeichert und zu Kontroll- und Systemsteuerungszwecken als Echogramme am Schiffsmonitor dargestellt. Durch die große Bandbreite und die schmale Schallkeule liefert das SBP-System ein Echogramm mit hohem Kontrast und ermöglicht eine maximale Schichtauflösung von wenigen Zentimetern.

\subsection{Postprocessing-Software}

Die Weiterverarbeitung der Messdaten, wie Filterung, Definition signifikanter akustischer Reflektoren oder Bohrkernüberlagerung, kann mit einer systemeigenen Software oder mit Produkten von Fremdherstellern erfolgen. Innomar bietet hierfür die systemeigene Software ISE (Interactive Sediment Layer Editor) an. Da Kongsberg über keine eigene Postprocessing-Software verfügt, wurden hierfür die Sub-Bottom-Module von Hypack sowie Sonarwiz eingesetzt. Die verwendeten Softwarepakete sind im Vergleich zu Highend-Produkten zur Auswertung reflexionsseismischer Daten, wie KINGDOM, Survey Engine Seismic+ oder Delph Seismic, auf die Grundfunktionalität der Verarbeitung von SBP-Daten reduziert.

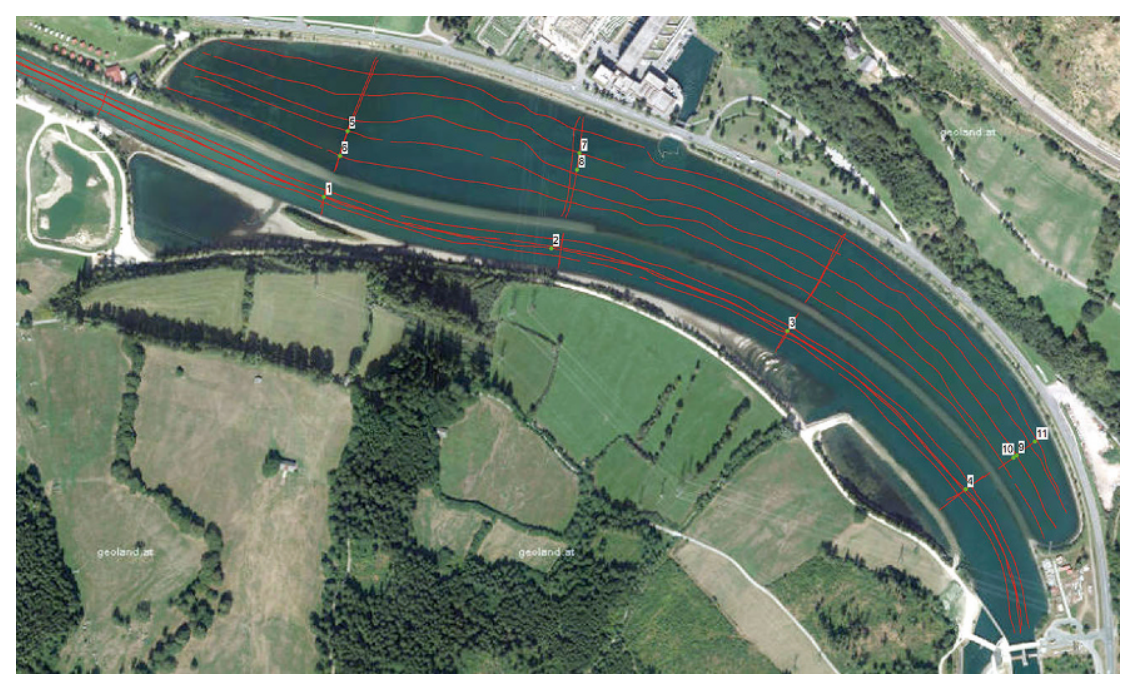

Abb. 2 Übersicht über die Messfahrten und Probenahmepunkte im Speicher Rottau

\section{Anwendungsbeispiel Speicherbecken Rottau}

\subsection{Projektgebiet}

Das Speicherbecken Rottau liegt im unteren Mölltal zwischen den Gemeinden Reißeck und Mühldorf. Dieses Becken ist Teil der Hauptstufe der Maltakraftwerksgruppe und dient dem Kraftwerk Rottau als Ausgleichsbecken, um auch den Einsatz im Pumpbetrieb zu ermöglichen. Von Westen nach Süden wird das Speicherbecken von einer sogenannten „Fließrinne“ der Möll durchzogen, welche durch unter der Wasseroberfläche liegende Leitdämme vom restlichen Becken getrennt wird. Am südlichen Ende des Beckens befindet sich eine Wehranlage, welche mittels Klappenregelung Spülungen des Stauraums ermöglicht. Aufgrund der stark reduzierten Fließgeschwindigkeiten bzw. stehender Wasserbereiche im
Speicherbecken kommt es zu teils erheblichen Feinsedimentablagerungen im gesamten Speicherbereich (Ausnahme Möllrinne, da hier regelmäßig gespült wird). Aufgrund dieser Charakteristik der Sedimentzusammensetzungen außerhalb der „Möllrinne“ waren hier sehr gute Rahmenbedingungen gegeben, die Möglichkeiten des SBP zur Detektion von Feinsedimentschichtungen auszutesten.

\subsection{Datengrundlage Bathymetrie und Aufnahmeplanung}

Als Planungsgrundlage für die Messungen wurden vom Betreiber VERBUND Tiefenprofildaten der jährlich durchgeführten Echolotungen (Monitoring) sowie ein aus einer Fächerecholotmessung 2015 stammendes digitales Geländemodell zur Verfügung gestellt. Auf dieser Basis erfolgte die Festlegung der Messprofile für die seismischen Mes-

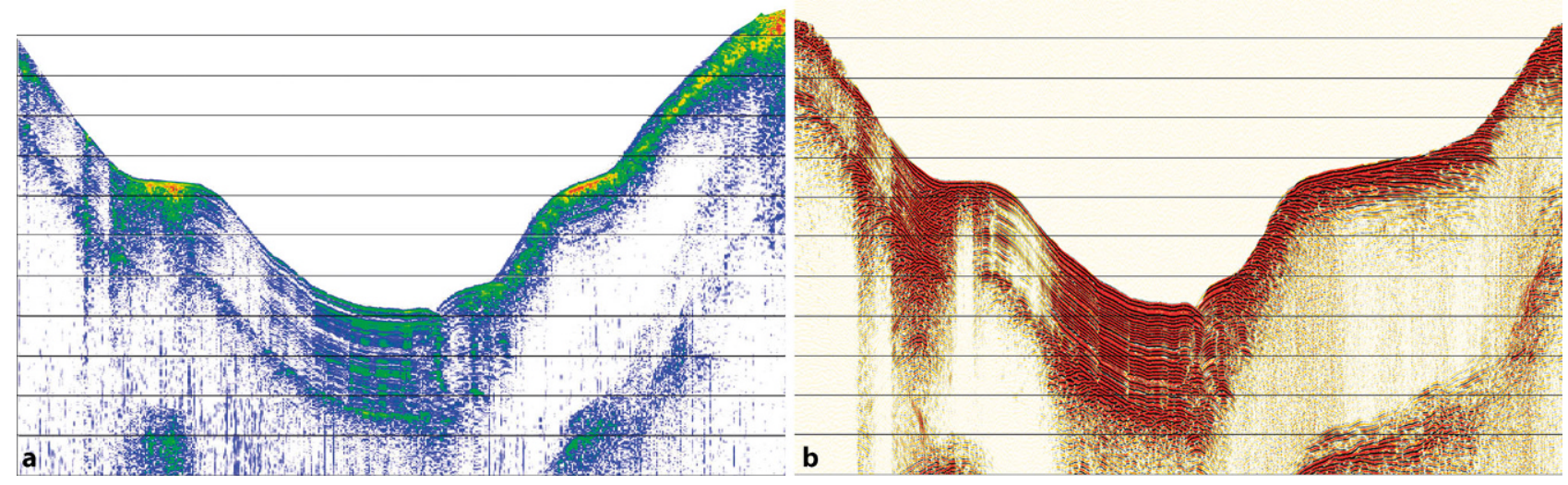

Abb. 3 Beide SBP-Systeme (a TOPAS; b SES2000) erzielten eine Eindringung von mehreren Metern 

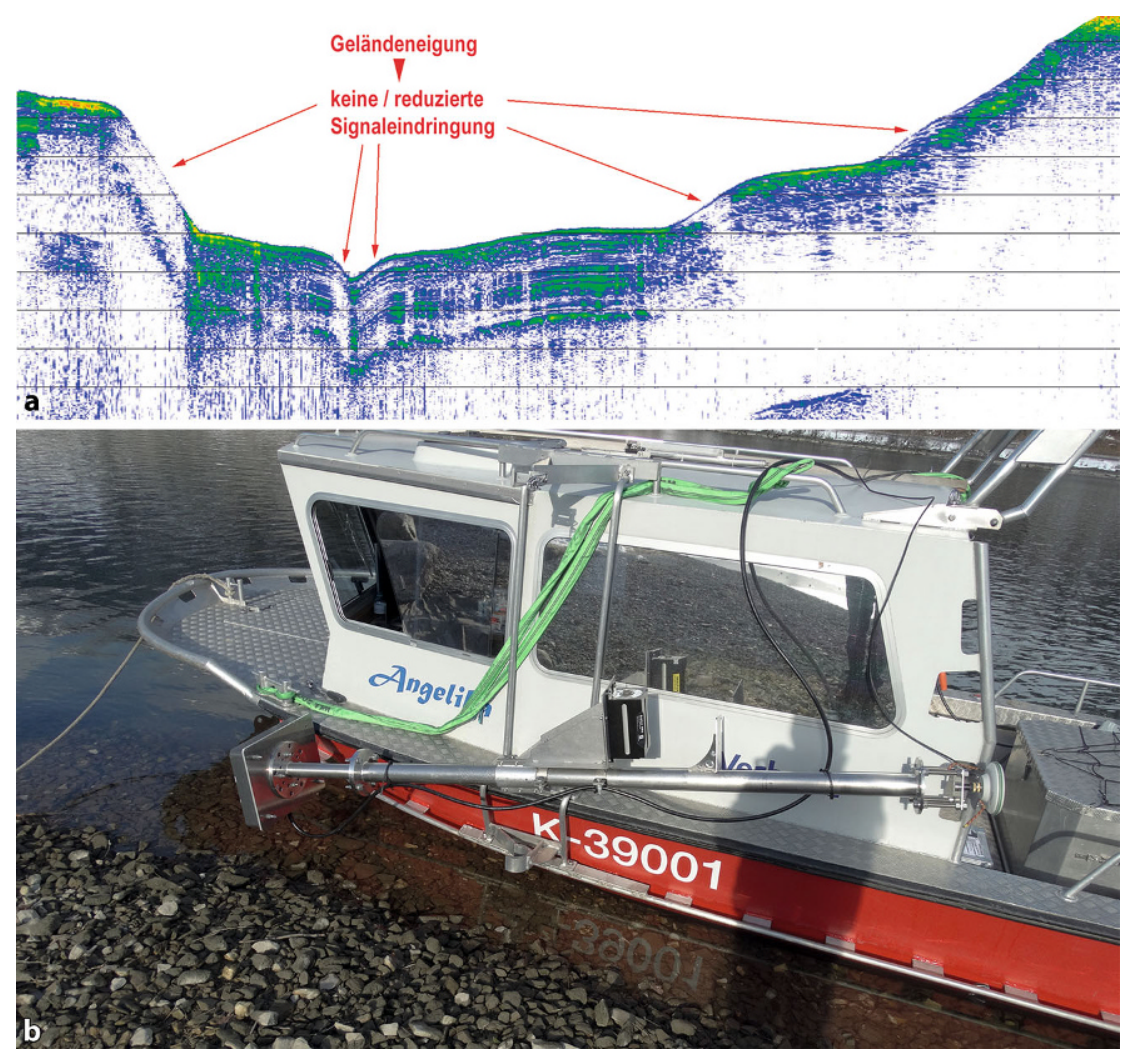

Abb. 4 a Geringe oder keine Reflexionen an steilen Geländeflächen, b Messboot mit Sub-Bottom-Profiler, Bewegungssensor und GNSS-Antenne

sungen. Die Längsprofile wurden direkt entlang sowie parallel zum zentralen Längsgraben (Möllrinne) und unter Einbindung morphologisch interessanter Sohlstrukturen des Ausgleichsbeckens festgelegt. Die Lage der Querprofile für die seismischen Messungen entspricht ebenfalls genau der Lage der Monitoring-Profile des VERBUND. Somit ergibt sich die Möglichkeit der Validierung des aus seismischen Daten ermittelten historischen Sedimentationsprozesses durch reale Messwerte aus dem jeweiligen Betrachtungszeitpunkt. Die Lage der Längs- und Querprofile ist in Abb. 2 ersichtlich.

\subsection{Sub-Bottom-Profiler-Messungen}

Im Februar 2018 fanden an drei aufeinanderfolgenden Tagen die Messungen mit den Sedimentecholoten Innomar SES2000 (8 kHz) und Kongsberg TOPAS PS 120 im Auffangbecken und in der Fließrinne statt. Die Messfahrten mit dem SES2000-System wurden entlang vordefinierter Profile (acht Längs- sowie vier Querprofile) durchgeführt (vgl. Abb. 2). Für die nachfolgende Messfahrt mit dem TOPAS-System wurde dann die reale Fahrspur der SES2000Messfahrt als Sollspur herangezogen. Als Messplattform diente das Vermessungsboot des VERBUND, welches mit einem zentimetergenauen RTK-GNSSPositionierungssystem und einem INSSystem ausgestattet ist, welches die Rollbewegungen mit einer Genauigkeit von $\pm 0,1^{\circ}$ erfasst (vgl. Abb. $4 \mathrm{~b}$ ). Damit kann die tatsächliche Position des einzelnen Messpunkts an der Gewässersohle mit einer Genauigkeit von wenigen Zentimetern bestimmt werden und Messergebnisse aus unterschiedlichen Messepochen können aufgrund ihrer präzisen Georeferenzierung miteinander verglichen werden. Beispiele für die Messungen beider verwendeter Systeme sind in Abb. 3 bzw. Abb. 4 ersichtlich.

\subsection{Sedimentbohrkerne}

\subsubsection{Kernbohrung}

Um die seismischen Daten geologisch richtig $\mathrm{zu}$ interpretieren, wurden für die Schichtbeschreibungen Messdaten benötigt. Diese sollten nach Möglichkeit im Projektgebiet gut verteilt sein und dabei auch alle Sedimentzusammensetzungen abdecken. Die Festlegung der konkreten Position, an denen Kernbohrungen durchgeführt werden sollten, erfolgte anhand der Messdaten der im Februar 2018 durchgeführten Sedimentecholotungen. Dabei wurden in den hochauflösenden Echogrammen jene Gebiete ausgewählt, welche neben den oben genannten Aspekten insbesondere einen auffälligen Schichtungsverlauf der Sedimentablagerungen zeigten.

Ende Juli 2019 wurden an elf Positionen im Ausgleichsbecken ungestörte Sedimentbohrkerne durch die Firma UWITEC entnommen. Vier dieser Bohrkerne waren in der sogenannten „Möllrinne“ situiert, die restlichen Kerne $(n=7)$ lagen im Beckenbereich nördlich des Leitdamms (Abb. 2). Über GPS-Navigation wurde die im Vorfeld definierte Position angesteuert, im Anschluss wurde die Arbeitsplattform (Katamaran) über drei Ankerpunkte fixiert.

Mit einem Hammer Corer wurden zwei Meter lange PVC-Rohre mit einem Innendurchmesser von $8,6 \mathrm{~cm}$ und einer Wandstärke von $2 \mathrm{~mm}$ in den Untergrund getrieben. Bei Erreichen der gewünschten Tiefe, des Beckengrunds oder grober Sedimentfraktionen (z.B. Kies) wurde das Einschlagen gestoppt und das Plexiglasrohr mit den darin befindlichen Sedimenten aus dem Wasser gezogen. Noch knapp unter der Wasseroberfläche wurde das Rohr mit einem Deckel dicht verschlossen und durch Bohren eines Lochs oberhalb der Sedimentoberfläche wurde das überschüssige Wasser im Rohr abgelassen. Nach einigen Stunden wurde das PVC-Rohr ca. $5-10 \mathrm{~cm}$ oberhalb der Sedimentoberfläche abgeschnitten. Der Bereich zwischen Sedimentoberfläche und PVC-Rohr-Oberkante wurde mit Steckschaum aufgefüllt, um eine nachträgliche Störung des Probenmaterials bei Transport bzw. Lagerung zu verhindern und mit einem Deckel dicht verschlossen. Die Anwendbarkeit dieser Methoden stößt an ihre Grenzen, sobald gröberes Material im Untergrund vorliegt (> Feinkies). Eine solche Schicht ist somit als limitierender Faktor in Bezug auf die Bohrkernlänge anzusehen (siehe Beispiel Kern 9 in Abb. 6 und 7).

\subsubsection{Analyse der Bohrkerne}

Korngrößenanalyse Die Korngrößenanalyse erfolgte durch Nasssiebung mit anschließender Sedimentationsanalyse 

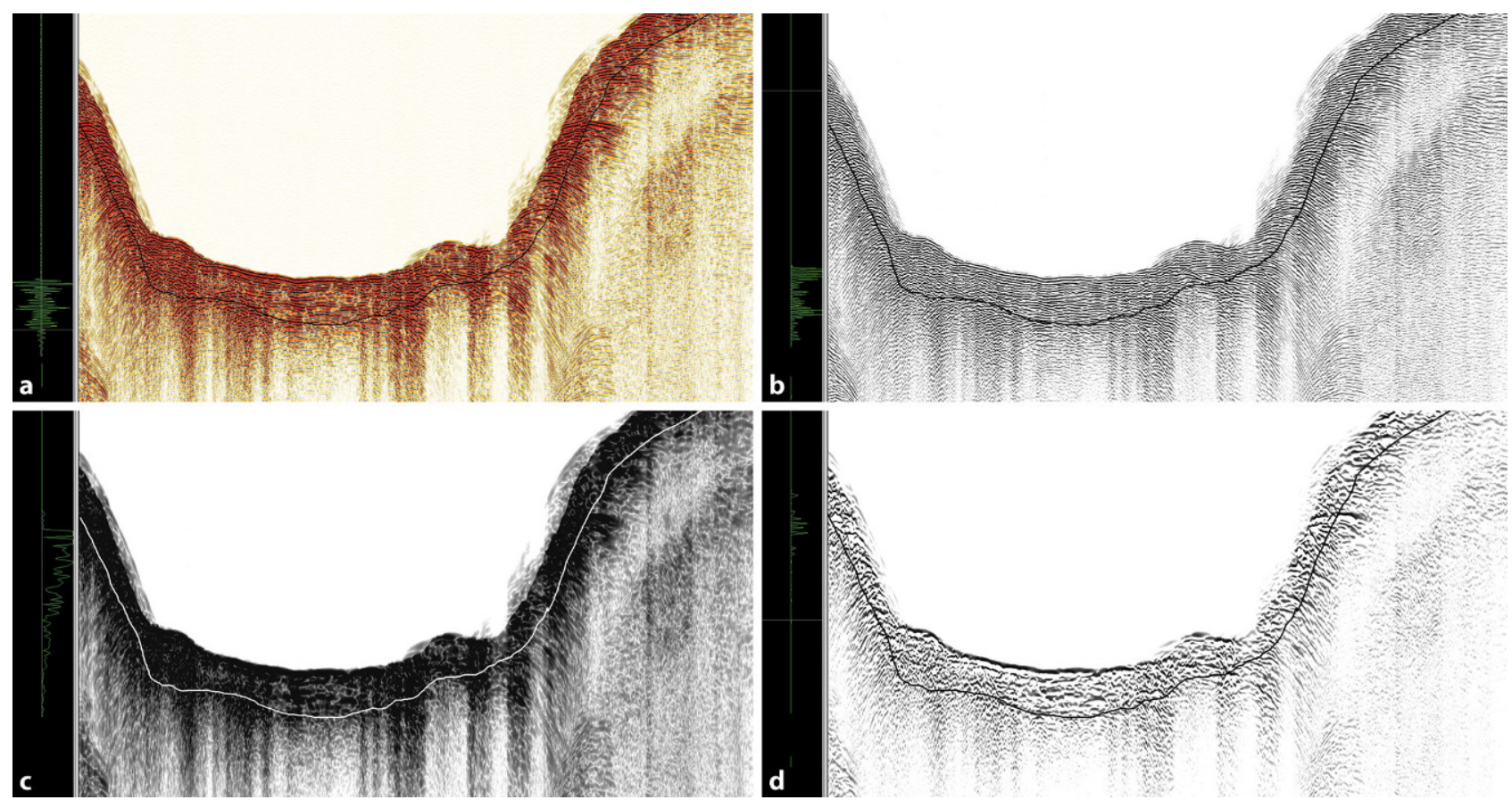

Abb. 5 Echogrammdarstellung der Reflexionssignale mit unterschiedlichen Algorithmen: a Amplitude, b Gradient der Amplitude, c Amplitude, Einhüllende, d Gradient, Einhüllende

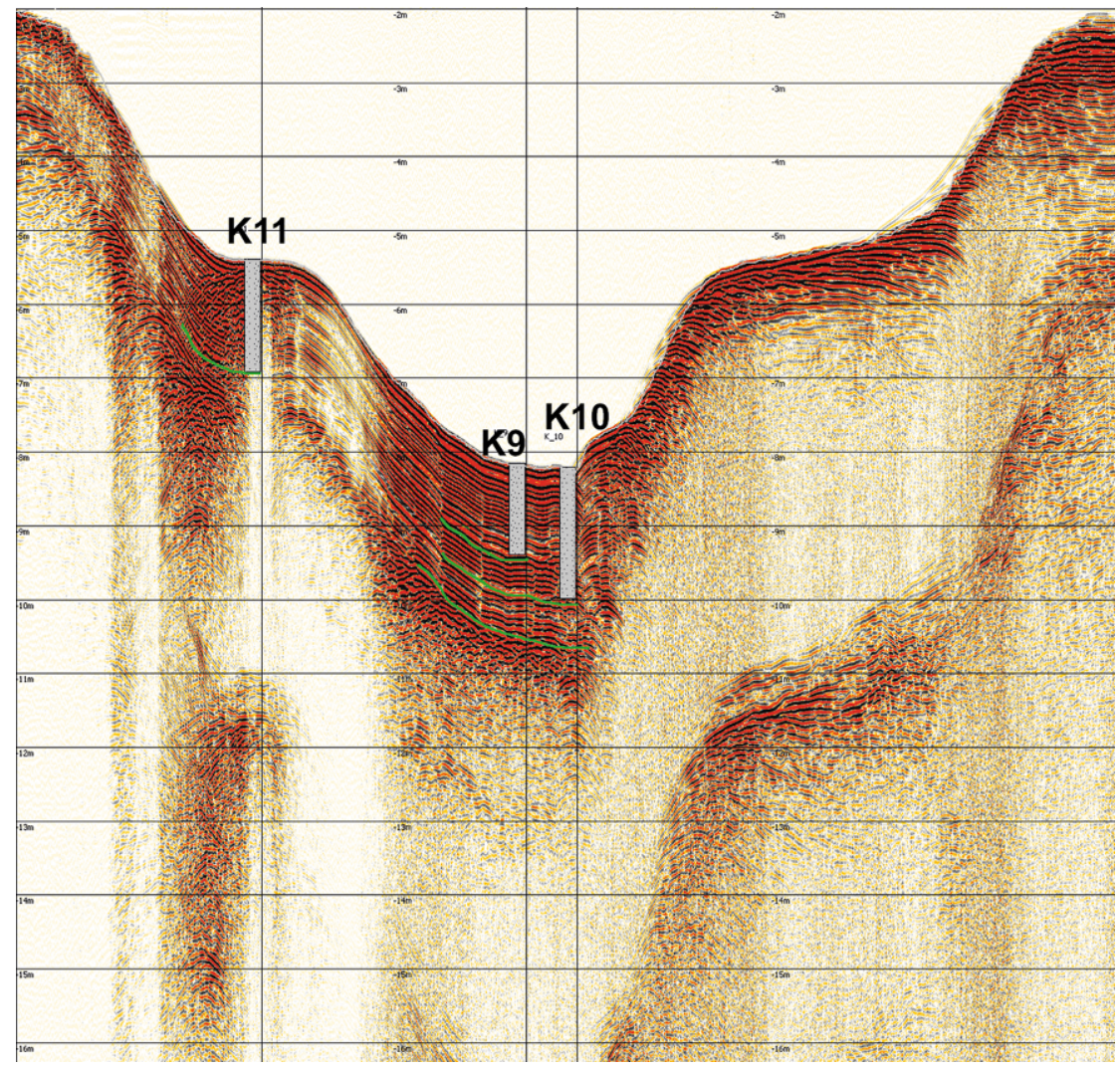

Abb. 6 Kombination von Echogramm und Sedimentkerndaten im Querprofil 27. Die grüne Linie kennzeichnet ausgeprägte Reflexionshorizonte mittels Sedigraph der Firma Micromeritics. Im Vorfeld wurde die zu siebende Probe über mehrere Tage mit Wasserstoffperoxid versetzt; dies dient zur Dispergierung und Zerstörung der organischen Substanz. Nach Beendigung der Reaktion wurde überschüssiges Wasserstoffperoxid durch Auskochen im Wasserbad entfernt, außerdem erfolgte eine Ultraschallbehandlung des Probenmaterials unmittelbar vor dem Sieben. Der verwendete Siebturm bestand aus Sieben mit den Maschenweiten $2000 \mu \mathrm{m}, 630 \mu \mathrm{m}, 200 \mu \mathrm{m}, 63 \mu \mathrm{m}$ und $20 \mu \mathrm{m}$. Der Siebdurchgang $<20 \mu \mathrm{m}$ wurde aufgefangen und das vorhandene Wasser durch Eindampfen reduziert, die Siebrückstände wurden bis zur Gewichtskonstanz getrocknet und gewogen. Eine Teilprobe der Fraktion $<20 \mu \mathrm{m}$ wurde für die weiterführende Sedimentationsanalyse eingedickt, mit einer Natriumpolyphosphatlösung $(0,05 \%)$ versetzt und im Sedigraph gemessen. Für die Ermittlung der Korngrößenverteilung wurden im Anschluss die Ergebnisse der Siebanalyse und der Sedimentationsanalyse herangezogen. Lagerungsdichte Die Bestimmung der Lagerungsdichte erfolgte über das Verhältnis der Masse des vorliegenden Materials in Bezug auf ein definiertes Volumen. Hierzu wurden Stechzylinderproben aus vordefinierten Schichten des Sedimentbohrkerns entnom- 


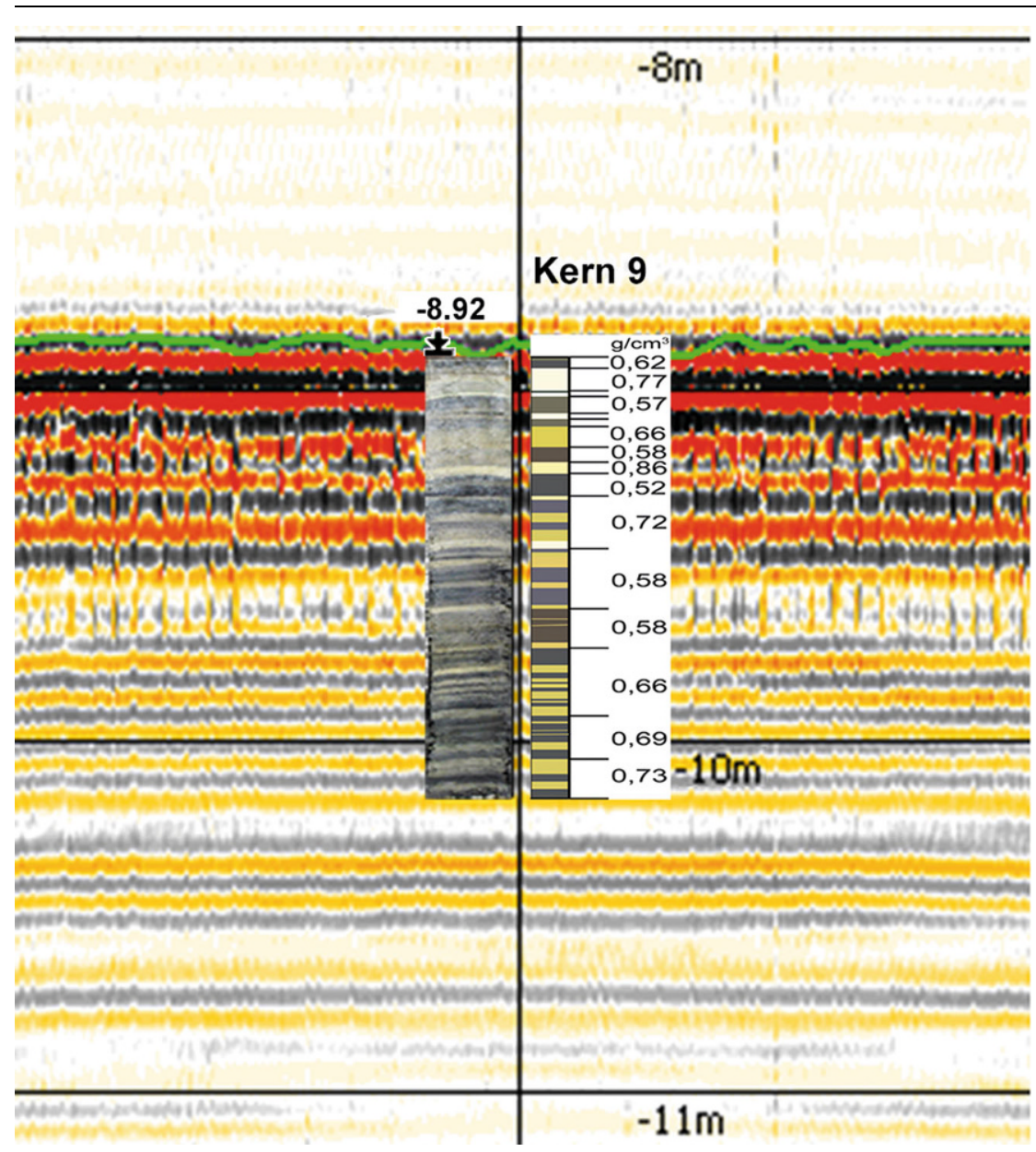

Abb. 7 Seismische Reflexionshorizonte (Echogramm) und Sedimentkerndaten (Lagerungsdichte) am Probenpunkt 9

men und bis zur Gewichtskonstanz getrocknet. Setzt man nun die Masse des getrockneten Materials in Verhältnis zum definierten Volumen des Stechzylinders, erhält man die Lagerungsdichte in $\mathrm{g} / \mathrm{m}^{3}$.

\subsection{Verknüpfung seismischer und Kernbohrungsdaten}

Die Verknüpfung der resultierenden Sedimentkerndaten mit SBP-Echogramminformationen erfolgte primär mit dem Programm ISE der Firma Innomar, da die Ergebnisse der InnomarMessungen auch über die beste Auflösung der akustischen Reflexionen des Sedimentkörpers (Sohle) verfügten. Für eine optimale Echogrammdarstellung der Reflexionshorizonte, durch welche Strukturen von Interesse deutlicher hervorgehoben werden, stehen diverse Signalfilter, Demodulationsmethoden (Roh, Einhüllende, Einhüllende+Filter) sowie Algorithmen (Echoamplitude,
PAS-Systems wurden die Aufnahmen in das standardisierte Format (SEG-Y) für geophysikalische Daten überführt und im Programm SonarWiz 7 der Firma Chesapeake sowie HYPACK Sub Bottom weiterbearbeitet.

Ein beispielhaftes Ergebnis für einen Vergleich findet sich in Abb. 6 und 7. Erste Analysen im vorderen Ausgleichsbeckenbereich zeigen einen Zusammenhang zwischen Korngrößen beziehungsweise Lagerungsdichteunterschieden und der Ausprägung signifikanter akustischer Reflektoren (Abb. 7). Ganz besonders deutlich zeigt sich auch hier schon die später noch behandelte Reflexionseigenschaft von Kiesschichten, welche ausgeprägte Reflexionsschichten darstellen und oft auch das Ende der Sedimentkerne definieren (Abb. 6 und 7). Eine Schichtabgrenzung in manueller Form ist hier im Untersuchungsgebiet sehr gut möglich. Für eine automationsgestützte Auswertung über alle Profile wird an einer Verbesserung der Abgrenzung durch erweiterte Filterung und Preprozessierung der Daten gearbeitet.

\section{Anwendungsbeispiel Donau}

\subsection{Aufgabenstellung}

In Hinblick auf die Erforschung der Kiesmächtigkeit in der Donau wurden mit den oben besprochenen Systemen auch an zwei Teilabschnitten der Donau Sub-Bottom-Profiler-Messungen durchgeführt. Dabei stellt hier das Kiesbett den limitierenden Faktor (die besondere Herausforderung) für einen erfolgreichen Messeinsatz von SBP-Systemen dar. Sand und Kies neigen dazu, einen Großteil der akustischen Energie $\mathrm{zu}$ reflektieren und die verbleibende Energie zu absorbieren. Aus diesem Grund können interne Grenzschichtreflexionen und darunterliegende Sedimentschichten nur sehr schwer oder gar nicht identifiziert werden (Pickering und Hiscott 2015). Im Rahmen von Testmessungen sollte nun konkret untersucht werden, ob und inwieweit die unterschiedliche Kiesmächtigkeit der Donau eine Bestimmung von Schichtgrenzen mittels SBP-Systemen zulässt. Für die Festlegung von Messgebieten mit unterschiedlichem Deckaufbau der Flusssohle und als Referenz für die Analyse sowie Validierung der SBPMessergebnisse standen für die untere Donau Informationen aus Freeze-CoreProbennahmen zur Verfügung (siehe 


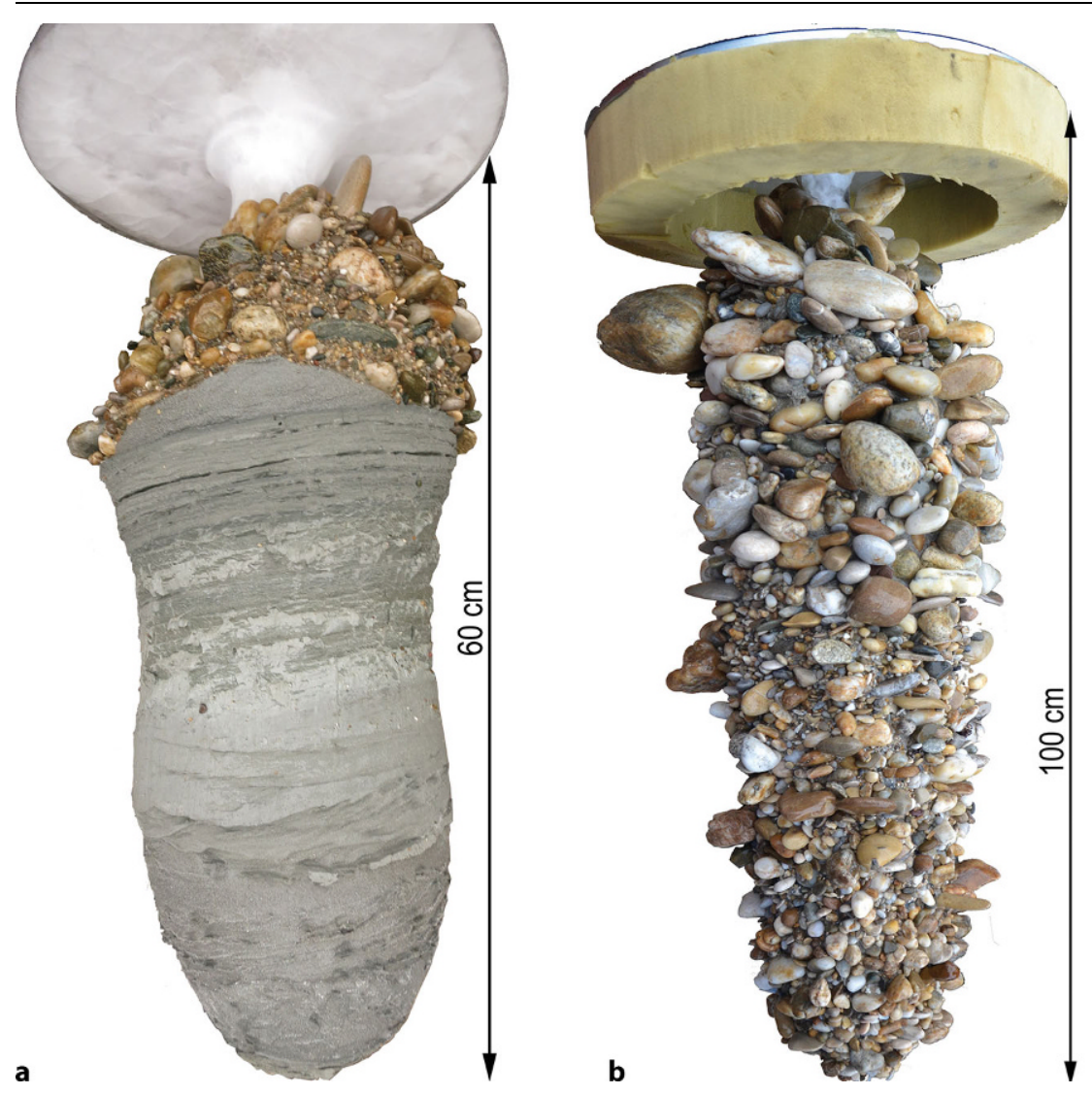

Abb. 8 Freeze Cores. (Liedermann et al. 2016)

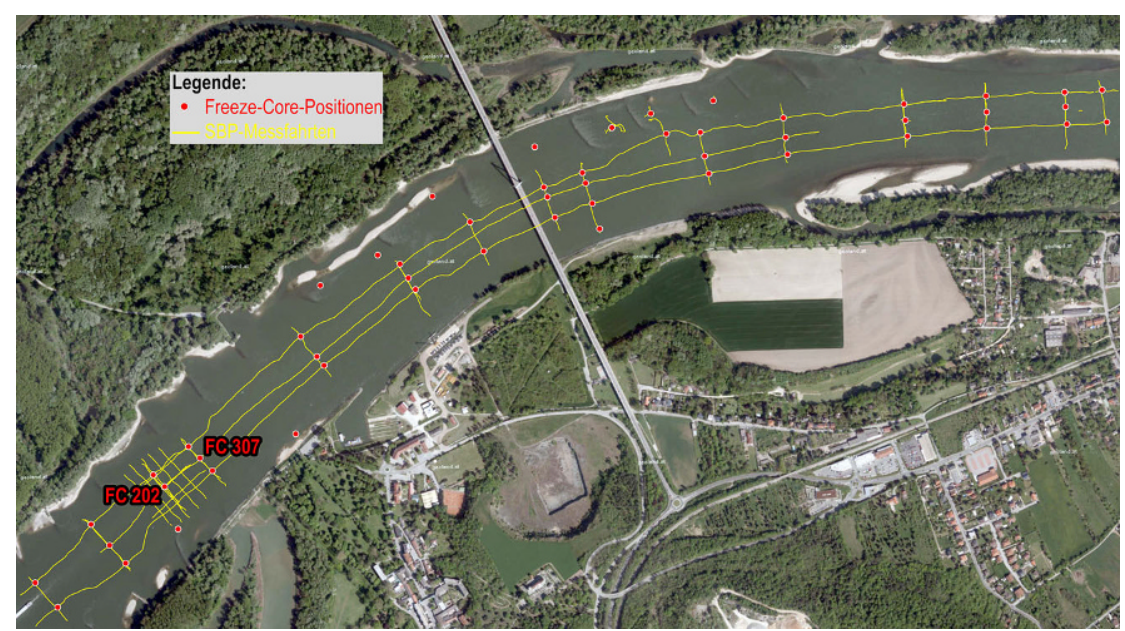

Abb. 9 Donau (Bad Deutsch-Altenburg) mit SBP-Messfahrten und Freeze-CorePositionen

Abschn. 4.2). Für den oberen Donauabschnitt wurden bei Hundsheim sowie Schönbühel jeweils zwei Kilometer Flusssohle ebenfalls durch Längs- und Querprofilfahrten aufgenommen.

\subsection{Freeze-Core-Daten}

Im Bereich zwischen Bad Deutsch-Altenburg und Hainburg wurden im November 2015 und 2017 vom Flussbett der Donau sogenannte „Freeze-Core“Kernproben entnommen (Habersack 2016; Liedermann et al. 2016). Bei dieser Methode wird von einer Schwimm- plattform aus ein bis zu 1,5m langes Edelstahlrohr (Durchmesser $4,5 \mathrm{~cm}$ ) mithilfe von Gewichten in den Untergrund eingeschlagen. Durch innenliegende Verteilungskanäle dringt Flüssigstickstoff in den Untergrund ein und führt zu raschem Anfrieren des umliegenden Sediments. Im genannten Untersuchungsgebiet wurden 52 Sedimentkerne mit einem Durchmesser von bis zu $30 \mathrm{~cm}$ und einer Kernlänge von bis $\mathrm{zu}$ einem Meter entnommen (Abb. 8 und 9).

\subsection{Sub-Bottom-Profiler-Messungen}

Die Fahrprofile für die SBP-Messungen wurden so gelegt, dass die Positionen der Freeze-Core-Proben einmal in Längsfahrt und mindestens einmal in Querfahrt übermessen wurden (Abb. 9). An ausgewählten Freeze-Core-Punkten (Nr. 202-203 und 306-307-308) erfolgte die Querprofilbefahrung in beide Richtungen. Damit können einerseits eventuelle Einflüsse der unterschiedlichen Fahrdynamik und anderseits eventuell vorkommende kontinuierliche Schichtungsverläufe im Untergrund anhand von Mehrfachfahrten analysiert werden.

Die SBP-Messungen mit dem Innomar SES-2000 wurden Anfang Dezember 2017, jene mit dem Kongsberg TOPAS im Februar 2018 durchgeführt. Als Messplattform diente dabei das Vermessungsboot Epsilon der via donau, welches dank seiner integrierten „survey grade" GNSS- und INS-Einheit auch gleich zentimetergenaue Positions- und präzise Bewegungsdaten an das SBPMesssystem lieferte. Die Montage der Echolotschwinger erfolgte mittels eines multifunktionalen, für mobile Bootseinsätze ausgelegten Aluträgersystems. Die Messfahrten in den einzelnen Projektgebieten konnten jeweils an einem Tag realisiert werden.

\subsection{Analyse und Ergebnis}

Aufgrund des Kiesaufbaus der Sohldeckschicht (vgl. Abb. 8) lag die erzielte Eindringtiefe in den Sedimentkörper zwischen wenigen Zentimetern und maximal 2 Metern. Dabei lieferten beide Systeme sehr ähnliche Ergebnisse (vgl. Abb. 10).

In sämtlichen Profilen zeigen sich fast durchgängig extrem starke Oberflächenreflexionen mit einer sehr ausgeprägten Internreflexion in der Deckschicht. Im Vergleich zu Rottau tritt 

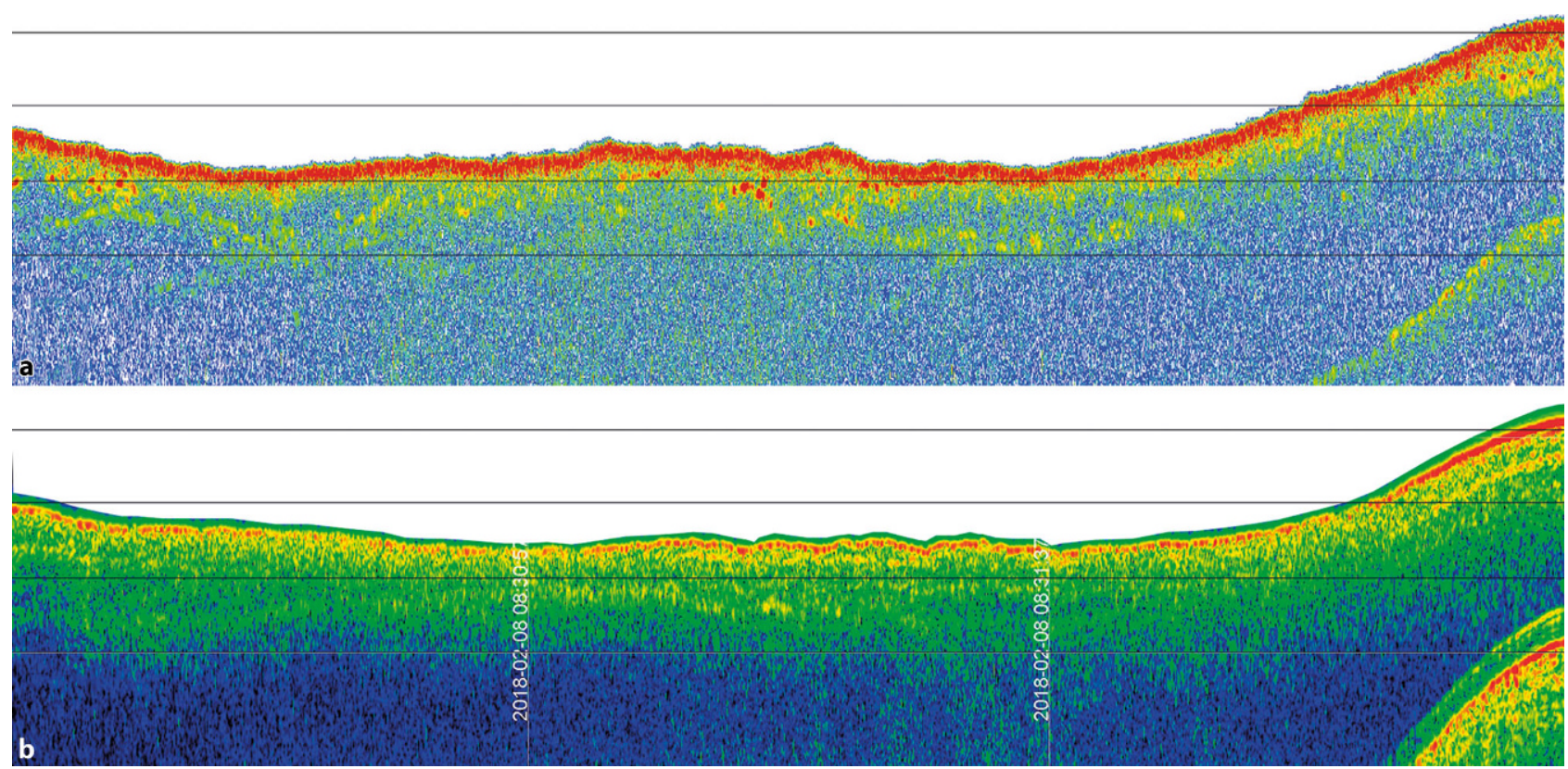

Abb. 10 SBP-Querprofil des kiesbedeckten Donau-Flussbetts: a SES2000, b TOPAS, starke Oberflächen- und Internreflexionen in der Deckschicht. Resteindringung bis etwa $2 \mathrm{~m}$
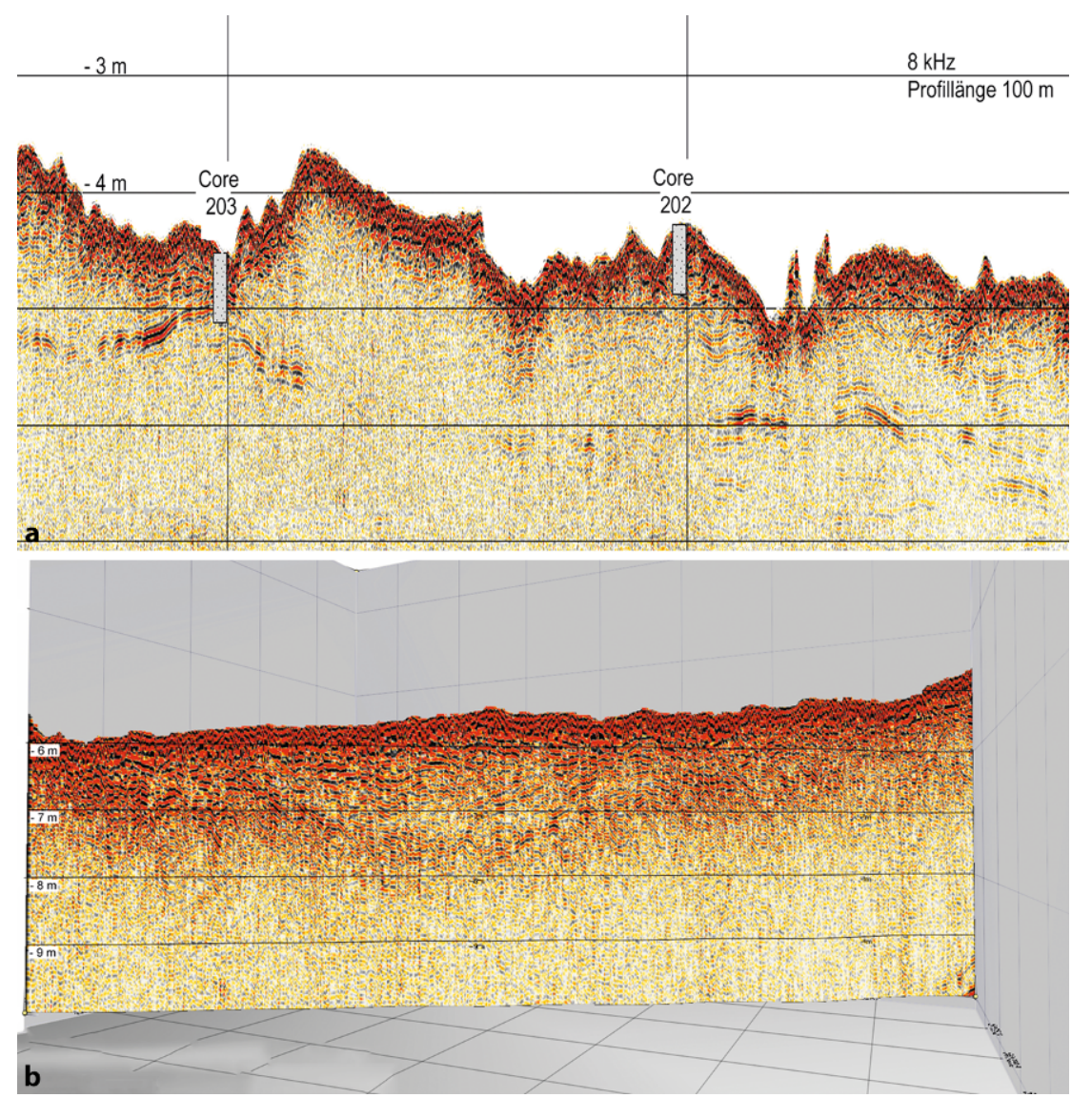

Abb. 11 SES2000-SBP-Echogramme der beiden Untersuchungsgebiete mit starken Internreflexionen in der Deckschicht (Kies) und eindeutigen akustischen Reflektoren in tieferen Zonen des Sedimentkörpers des Flussbetts dabei ein um etwa $10-15 \mathrm{~dB}$ erhöhtes Hintergrundrauschen auf. An einigen wenigen Stellen in den Profilen sind auch in tieferen Lagen akustische Reflektoren $\mathrm{zu}$ detektieren, wie etwa im Querprofil zwischen den beiden FreezeCores 202-203 (Abb. 11a). In diesem Bereich beträgt die Dicke der Kiesschicht nur etwa $10-20 \mathrm{~cm}$. Darunter befindet sich dann eine ausgeprägte Schicht aus tertiärem Feinmaterial/Tegel von mindestens $80 \mathrm{~cm}$ Stärke (maximale FreezeCore-Länge). Eine Abgrenzung der dünnen Kiesauflage zum anschließenden Tegelkörper ist hier mit der gewählten Messfrequenz von $8 \mathrm{kHz}$ und der starken Internreflexionen im Kieskörper nicht möglich. Und obwohl hier mit den niedrigfrequenten akustischen Wellen der SBP-Systeme die Kiesschicht durchdrungen und die tieferliegenden akustischen Reflektoren registriert werden konnten, ist eine klar ausgeprägte Begrenzung des Tertiärs nicht eindeutig über den gesamten Profilverlauf erkennbar (Abb. 11a). Jedoch scheinen im überwiegenden Teil der Messprofile Teilabschnitte mit klar abgrenzbaren Reflektoren auf, welche durch schwächer ausgeprägte Reflektoren verbunden sind. Beispielhaft sei hierfür auf die Ergebnisse der Messungen im zweiten Untersuchungsgebiet bei Hundsheim in der Wachau verwiesen (Abb. 11b). 


\section{Ausblick}

Die zukünftigen Forschungen werden sich der weiteren Analyse in Hinblick auf die Übereinstimmung akustischer Reflexionshorizonte und sedimentologisch/geologischer Schichtungscharakteristik widmen, mit dem Ziel, Auskunft darüber geben zu können, inwieweit seismische Verfahren für die Erfassung der Eigenschaften von abgelagerten Sedimenten in Stauräumen und großen Flüssen (Wasserstraßen) eingesetzt werden können, um in Zukunft ein verbessertes Sedimentmanagement erzielen bzw. umsetzen zu können. Es ist geplant, im BOKU-Forschungsgerinne systematische Versuche mit klar definierten Schichten verschiedener Korngrößen und Materialdichte etc. vorzunehmen. Des Weiteren soll versucht werden, die bei den aktuell getesteten Systemen festgestellten, systembedingten Einschränkungen durch weitere Tests mit alternativen geophysikalischen Methoden zu überwinden. Dabei handelt es sich neben den Methoden der Seismik (siehe Tab. 1) auch um bereits im kleinen Rahmen erprobte Methoden der Geoelektrik und des Georadars.

Danksagung Besonderer Dank gebührt den Firmen Kongsberg Maritime
Deutschland und Kongsberg Norwegen für die Bereitstellung ihres Messsystems sowie ihrer Experten (J. Dybedal \& G. Neland) für die Messarbeiten und J. Lowag von Innomar für die Unterstützung bei der Filterung und Analyse der Full-Waveform-Messdaten. Des Weiteren danken wir den Studierenden der Universität für Bodenkultur Wien S. Wagner und T. Reiter für ihre Messunterstützung (Echolotung und GNSSPositionierung) während der Sedimentkernbohrungen in Rottau und Marcel Liedermann für die Bereitstellung der Freeze-Core-Daten der Donau.

Förderung Wir bedanken uns für die finanzielle Unterstützung durch die Christian-Doppler-Forschungsgesellschaft, das Bundesministerium für Digitalisierung und Wirtschaftsstandort und die Nationalstiftung für Forschung, Technologie und Entwicklung. Darüber hinaus möchten wir den Unternehmenspartnern Verein für Ökologie und Umweltforschung (VÖU), viadonau, Andritz AG und Voith $\mathrm{GmbH}$ für die Förderung der Forschungsarbeiten im CD-Labor danken.

Funding Open access funding provided by University of Natural Resources and Life Sciences Vienna (BOKU).
Open Access Dieser Artikel wird unter der Creative Commons Namensnennung 4.0 International Lizenz veröffentlicht, welche die Nutzung, Vervielfältigung, Bearbeitung, Verbreitung und Wiedergabe in jeglichem Medium und Format erlaubt, sofern Sie den/die ursprünglichen Autor(en) und die Quelle ordnungsgemäß nennen, einen Link zur Creative Commons Lizenz beifügen und angeben, ob Änderungen vorgenommen wurden.

Die in diesem Artikel enthaltenen Bilder und sonstiges Drittmaterial unterliegen ebenfalls der genannten Creative Commons Lizenz, sofern sich aus der Abbildungslegende nichts anderes ergibt. Sofern das betreffende Material nicht unter der genannten Creative Commons Lizenz steht und die betreffende Handlung nicht nach gesetzlichen Vorschriften erlaubt ist, ist für die oben aufgeführten Weiterverwendungen des Materials die Einwilligung des jeweiligen Rechteinhabers einzuholen.

Weitere Details zur Lizenz entnehmen Sie bitte der Lizenzinformation auf http://creativecommons.org/licenses/ by/4.0/deed.de.
Baran, R., Dobler, W., Steinbacher, F., Ritter M., Niederwieser, M., Benger, W., \& Aufleger, M. (2013): Verschneidung von Airborne Hydromapping und Fächerecholotvermessung be Rheinfelden. WasserWirtschaft - Fachzeitschrift für Wasser und Umwelttechnik, 18-25. https:// doi.org/10.1365/s35147-013-0711-9

Bock, N., Gökler, G., Reindl, R., Reingruber, J., Schmalfuß, R., Badura, H., ... \& Spreitzer, R. (2019): Feststoffmanagement bei Wasserkraftanlagen in Österreich. Österreichische Wasserund Abfallwirtschaft, 71(3-4), 125-136.

Buchta, R. (2019): Entwicklung eines Ziel-und Bewertungssystems zur Schaffung nachhaltige naturnaher Strukturen in großen sandgeprägten Flüssen des norddeutschen Tieflandes.

Chapman, D. V. (Ed.). (1996): Water quality assessments: a guide to the use of biota, sediments, and water in environmental monitoring. Dang, T. H., Coynel, A., Orange, D., Blanc, G. Etcheber, H., \& Le, L. A. (2010): Long-term monitoring (1960-2008) of the river-sediment transport in the Red River Watershed (Vietnam). Temporal variability and dam-reservoir impact. Science of the Total Environment, 408(20), 4654-4664.

De Araujo, J. C., Güntner, A., \& Bronstert, A (2006): Loss of reservoir volume by sediment deposition and its impact on water availability in semiarid Brazil. Hydrological Sciences Journal, 51(1), 157-170.

Faghihirad, S., Lin, B., \& Falconer, R. A. (2015) Application of a 3D layer integrated numerical model of flow and sediment transport processes to a reservoir. Water, 7(10), 5239-5257.

Foster, I. D. L., \& Walling, D. E. (1994): Using reservoir deposits to reconstruct changing sediment yields and sources in the catchment of the Old Mill Reservoir, South Devon, UK, over the past 50 years. Hydrological Sciences Journal, 39(4), 347-368.

Habersack, H. (2016): Wasserbau, Schifffahrt und Ökologie an der Donau - Pilotprojekt Bad Deutsch-Altenburg. Österreichische Wasserund Abfallwirtschaft, 68, 190-192.

Hauer, C., Keckeis, H., Tritthart, M., Liedermann, M., \& Habersack, H. (2012): Optimierung der Habitatmodellierung an großen Fließgewässern unter Berücksichtigung von flussmorphologischen Prozessen auf unterschiedlichen Skalen. Österreichische Wasser-und Abfallwirtschaft, 64(11-12), 553-563.

Hauer, C., Wagner, B., Aigner, J., Holzapfel, P., Flödl, P., Liedermann, M., ... \& Haimann, M. (2018): State of the art, shortcomings and future challenges for a sustainable sediment management in hydropower: A review. Renewable and Sustainable Energy Reviews, 98, 40-55.
Haun, S., Kjærås, H., Løvfall, S., \& Olsen, N. R. B. (2013): Three-dimensional measurements and numerical modelling of suspended sediments in a hydropower reservoir. Journal of hydrology, 479, 180-188.

Habersack, H., Liedermann, M., Tritthart, M. Hauer, C., Klösch, M., Klasz, G., Hengl, M. (2012): Maßnahmen für einen modernen Flussbau betreffend Sohlstabilisierung und Flussrückbau - Granulometrische Sohlverbesserung, Buhnenoptimierung, Uferrückbau und Gewässervernetzung. Österreichische Wasser- und Abfallwirtschaft, 64, 571-581.

Heine, E. (2017): Hydroakustisches Monitoring des Ausläufers des Gschliefgrabenfächers im Traunsee, in: Lienhart, W. (Ed.), Ingenieurvermessung 17: Beiträge zum 18. Internationalen Ingenieurvermessungskurs Graz, 2017. Vde Verlag, Berlin, pp. 363-371.

Heine, E., Weidinger, J.T., Götz, J. (2016): Geologisch-geomorphologische Untersuchungen des subaquatischen Bereichs von Erdströmen in den Traunsee (OÖ) unter Anwendung von Fächerecholot und parametrischem Sedimentecholot. VGI: österreichische Zeitschrift für Vermessung \& Geoinformation 104, 25-37.

Hilbe, M., Anselmetti, F.S., Eilertsen, R.S., Hansen, L., Wildi, W. (2011): Subaqueous morphology of Lake Lucerne (Central Switzerland): implications for mass movements and glacial history. 


\section{Originalarbeit}

Swiss J Geosci 104, 425-443. https://doi.org/10. 1007/s00015-011-0083-z

Liedermann, M., Gmeiner, P., Glas, M., Tritthart, M., \& Habersack, H. (2016): Funktionalität der getesteten flussbaulichen Maßnahmen im Pilotprojekt Bad Deutsch-Altenburg. Österreichische Wasser-und Abfallwirtschaft, 68(5-6), 217-225

Mahmood, K., \& Mundial, B. (1987): Reservoir sedimentation: impact, extent, and mitigation. McIntyre, S. C. (1993): Reservoir sedimentation rates linked to long term changes in agricultural land use 1. JAWRA Journal of the American Water Resources Association, 29(3), 487-495.

Missiaen, T., Slob, E., Donselaar, M.E. (2008): Comparing different shallow geophysical methods in a tidal estuary, Verdronken Land van Saeftinge, Western Scheldt, the Netherlands. Netherlands Journal of Geosciences - Geologie en Mijnbouw 87, 151-164.

Navas, A., Valero-Garcés, B., Gaspar, L., \& Machín, J. (2009): Reconstructing the history of sediment accumulation in the Yesa reservoir: an approach for management of mountain reservoirs. Lake and Reservoir Management, 25(1), $15-27$.

Pickering, K., Hiscott, R. (2015): Seismic facies. In: Deep Marine Systems: Processes, Deposits,
Environments, Tectonics and Sedimentation. John Wiley \& Sons, New Jersey, pp. 62-111.

Ramsay, P. (2017): Sub-bottom Profiling Acquisition Techniques in HYPACK. In: Technical Notes; Hypack Training Presentations. http:// www.hypack.com/File\%20Library/Resource \%20Library/ Technical\%20Notes/Sub-bottomProfiling-Acquisition-Techniques-in-HYPACK.pdf. Zugriff: 23.4.2020

Schleiss, A. J., Franca, M. J., Juez, C., \& De Cesare, G. (2016): Reservoir sedimentation. Journal of Hydraulic Research, 54(6), 595-614. Schnabel, C., Naumann, M., Reinhardt, L. (2014): Bearbeitung und Interpretation hochauflösender Reflexionsseismik aus dem deutschen Nordsee-Sektor, Geopotenzial Deutsche Nordsee. Bundesanstalt für Geowissenschaften und Rohstoffe und Bundesamt für Seeschifffahrt und Hydrographie, Hamburg.

Stoker, M.S., Pheasant, J.B., Josenhans, H. (1997): Seismic Methods and Interpretation, in: Davies, T.A., Bell, T., Cooper, A.K., Josenhans, H., Polyak, L., Solheim, A., Stoker, M.S., Stravers, J.A. (Eds.), Glaciated Continental Margins: An Atlas of Acoustic Images. Springer Netherlands, Dordrecht, pp. 9-26. https://doi.org/10.1007/ 978-94-011-5820-6_2
Strasser, M., Berberich, T., Fabbri, S., Hilbe, M., Huang, J.-J.S., Lauterbach, S., Ortler, M., Rechschreiter, H., Brauer, A., Anselmetti, F., Kowarik, K. (2020): Geomorphology and event-stratigraphy of recent mass-movement processes in Lake Hallstatt (UNESCO World Heritage Cultura Landscape, Austria). Geological Society, London, Special Publications 500.

Tritthart, M., Flödl, P., Habersack, H., Hauer., C. (2020): HEM Impoundment-A Numerical Prediction Tool for the Water Framework Directive Assessment of Impounded River Reaches. accepted paper in WATER.

Tritthart, M., Liedermann, M., Klösch, M., \& Habersack, H. (2012): Innovationen in der Modellierung von Sedimenttransport und Morphodynamik basierend auf dem Simulationsmodell iSed. Österreichische Wasser- und Abfallwirtschaft, 64(11-12), 544-552.

Hinweis des Verlags Der Verlag bleibt in Hinblick auf geografische Zuordnungen und Gebietsbezeichnungen in veröffentlichten Karten und Institutsadressen neutral. 